\title{
Effect of mutualistic and antagonistic bees on floral resources and pollination of a savanna shrub ${ }^{\text {is }}$
}

\author{
Marília Monteiro Quinalha ${ }^{\mathrm{a}}$, Anselmo Nogueira ${ }^{\mathrm{a}, \mathrm{b}}$, Gisela Ferreirac ${ }^{\mathrm{c}}$, Elza Guimarães ${ }^{\mathrm{c}, *}$ \\ a Graduation Program in Biological Sciences (Botany), Institute of Biosciences, UNESP - Univ Estadual Paulista, Botucatu, SP, Brazil \\ b Centro de Ciências Naturais e Humanas, Universidade Federal do ABC, São Bernardo do Campo, SP, Brazil \\ ${ }^{c}$ Departament of Botany, Institute of Biosciences, UNESP - Univ Estadual Paulista, Botucatu, SP, Brazil
}

\section{A R T I C L E I N F O}

\section{Article history:}

Received 24 May 2016

Received in revised form 26 August 2016

Accepted 30 August 2016

Edited by S.D. Johnson

Available online 6 September 2016

\section{Keywords:}

Bignoniaceae

Cheaters

Jacaranda caroba

Nectar

Pollen

Pollinators

\begin{abstract}
A B S T R A C T
Since Darwin, cheaters have been described in plant-pollinator mutualisms. Bignoniaceae species have a wide interaction network with floral visitors, and most of those interactions are established with cheaters. Thus, our objective was to determine which role each floral visitor plays in a system composed by bees and a Bignoniaceae savanna species. So, here we described the bees' behaviour and defined experimentally who are the mutualists and cheaters, we described the temporal sequence of interactions, quantified pollen and nectar removal, and checked for the potential effect of robbery damages on pollinator behaviour. Pollinators visited a small number of flowers, mainly in the early morning, while the most frequent cheaters (robbers and thieves) visited the flowers throughout the day, increasing visitation at midmorning, when pollinators had already visited the flowers. We considered medium-sized bees as pollinators, small bees acted only as cheaters, not contributing to seed production. Pollen thieves reduced the amount of male gametes available for pollination, while nectar thieves and robbers were associated with nectar depletion. We did not find any significant difference in the number of pollen grains deposited on the stigmas of flowers with and without robbery damages. In conclusion, when pollinators visit $J$. caroba flowers in the early morning, there might be a smaller competition pressure among visitors due to the greater amount of resources available, especially in recently opened flowers, and due to the low frequency of cheaters visits, which probably prevents a major negative impact of cheaters on pollination and plant reproduction in this species.
\end{abstract}

(c) 2016 Elsevier GmbH. All rights reserved.

\section{Introduction}

Floral communicative traits, such as scent (Vogel, 1990) and flower colours (Chittka and Thomson, 2001) may attract animals with similar sensory abilities (Schaefer and Ruxton, 2011). However, these visual cues and signals do not attract exclusively mutualistic animals searching for floral rewards, so in nature, floral resources are frequently exploited by a variable range of animals. Indeed, plant-pollinator mutualisms are cooperative interactions mediated by floral resources that can be susceptible to exploitative species acting as cheaters, without providing benefits to plants (Darwin, 1841; Thompson, 1982; Bronstein, 1994).

\footnotetext{
is This article is part of a special feature entitled: "Patterns and mechanisms in plant-pollinator interactions" published at FLORA volume 232, 2017.

* Corresponding author.

E-mail addresses: marilia_quinalha@yahoo.com.br (M.M. Quinalha), anselmoeco@yahoo.com.br (A. Nogueira),gisela@ibb.unesp.br (G. Ferreira), eguimaraes@ibb.unesp.br (E. Guimarães).
}

Cheaters can be classified in different ways due to their behaviour and impact on plants. In particular, thieves are visitors that enter the flower tube, by the same opening used by pollinators to access the floral resources, without causing damage to the flower, but a mismatch of morphologies precludes pollination; while robbers can access floral resources only through damages performed to the floral structure (Inouye, 1980). In both cases, floral resources, like pollen and nectar, can be depleted without pollen transfer services. Additionally, the intensity of cheaters' impact on mutualistic systems may depend on the temporal sequence in which these interactions take place (Barker and Bronstein, 2016). For example, cheaters visiting flowers earlier than pollinators can decrease floral resources, reducing flower attractiveness, which may lead to changes in pollinator behaviour and, consequently, negatively affect plant reproduction (Hargreaves et al., 2009).

Nectar is the main floral reward in angiosperms, and it is essential in determining the interactions between flowers and their visitors, both pollinators and cheaters (Willmer, 2011). Nectar robbery and theft may cause various effects on plants' reproductive 
success (Maloof and Inouye, 2000; Irwin et al., 2010). The impact of nectar robbers on pollinator behaviour is supposed to differ from the impact caused by nectar thieves because, besides the nectar depletion caused by both groups, the robbers may create visual signs (damages) that could be recognized by pollinators.

On the other hand, pollen thieves are associated with the reduction of pollen that, in other situations, could be removed and dispersed by pollinators (Hargreaves, 2007; Hargreaves et al., 2009). It may lead to a reduction of the amount of male gametes available for fertilization, directly and negatively affecting plants' sexual reproduction (Carmo et al., 2004). In some Jacaranda species, small bees, generally described as pollen thieves, may occasionally touch flowers' reproductive structures during pollen collection manoeuvres (Guimarães et al., 2008; Maués et al., 2008; MiletPinheiro and Schlindwein, 2009). Indeed, it was evidenced, for other biological systems, that small bees may transfer pollen grains to the stigma and, thus, occasionally act as pollinators (Hargreaves et al., 2009; Ballantyne et al., 2015). However, the effectiveness of floral visitors has been based, for example, on the frequency of interactions, presence of pollen on the insect's body, pollen deposition on the stigma, and pollen tube development (Castro et al., 2013). So, an experimental approach that tests the role of small bees, previously classified as pollen thieves, on the sexual reproduction of these plant species is lacking.

In fact, the putative role of each floral visitor on seed production remains unclear for most systems. Bignoniaceae species, for example, have shown a rich and wide interaction network with floral visitors, with $75 \%$ of the interactions established with cheaters (Genini et al., 2010). However, the real impact of cheaters on plant pollination remains poorly understood, probably due to the complexity of this communication network. Thus, our objective was to determine which role each floral visitor plays in a system composed by a Bignoniaceae species, Jacaranda caroba, and the bees that visit its flowers. For this purpose, we described the behaviour of each floral visitor and we tested the effectiveness of all the bees that entered the flower tube on the production of viable seeds; we characterized the temporal sequence of interactions displayed by all bees that visited J. caroba flowers; we quantified pollen and nectar removal by pollinators and cheaters; and we verified the potential effect of nectar robbery damages to the corolla tube on pollen grains deposition. Specifically, we asked: (i) who acts as cheaters and as pollinators in this system; (ii) how is the visitation pattern of cheaters and pollinators throughout the day; (iii) do cheaters affect pollen and nectar availability to pollinators; (iv) does nectar robbery damage negatively affect pollen deposition on the stigma?

\section{Material and methods}

\subsection{Plant species and study site}

We selected Jacaranda caroba as the focal plant species because it is involved in a rich network of interactions, including several cheaters species acting as thieves and robbers, with only two bee species recognized as pollinators due to their morphology and behaviour by Guimarães et al. (2008). Jacaranda caroba is the accepted name and J. oxyphylla, as referred in Yanagizawa and Maimoni-Rodella (2007) and in Guimarães et al. (2008), is currently treated as synonym (The plant list, 2013).

Jacaranda caroba (Vell.) DC. (Bignoniaceae) is a plant species endemic to Brazil that occurs mainly in cerrado lato sensu and shows a habit varying from shrub to small trees $(0.7-3.0 \mathrm{~m})$ (Gentry and Morawetz, 1992). This species is characterized by opposite phyllotaxis and bipinnate composed leaves; panicle inflorescence; tubular-campanulate flowers with dorsoventrally flat corollas, varying from light to dark purple; the reproductive structures are located in the centre of the flower tube; the androecium has didynamous stamens, with dithecate anthers and one long glandular staminode; the gynoeceum is composed of a pistil with a swollen ovary above the cylindrical nectary; fruits are elliptical capsules with membranous winged seeds that are wind-dispersed (Gentry and Morawetz, 1992). The specimens' vouchers are deposited in the Herbarium BOTU "Irina Delanova de Gemtchujnicov" (voucher numbers 32538, 32539 and 32540).

This study was conducted at the "Estação Ecológica de Santa Bárbara" from the "Instituto Florestal do Estado de São Paulo", located in São Paulo state, Brazil (approximately between $22^{\circ} 46^{\prime}$ to $22^{\circ} 50^{\prime}$ S and $49^{\circ} 10^{\prime}$ to $49^{\circ} 15^{\prime} \mathrm{W}$ ). The reserve has 2712 ha of cerrado sensu lato and seasonal forest vegetation. The climate is seasonal with average temperatures of $16^{\circ} \mathrm{C}$ and $23^{\circ} \mathrm{C}$ in the coldest and hottest months, respectively. Annual rainfall varies from 1000 to $1300 \mathrm{~mm}$; the soil is usually deep, acidic and nutrient-poor (Melo and Durigan, 2011).

In the cerrado sensu stricto areas (savanna like vegetation) we mapped and labelled 230 individuals of $J$. caroba distributed in an area of approximately $8 \mathrm{~km}^{2}$. During the flowering period, we randomly sampled several subsets of these labelled plants to use in different types of further detailed experiments.

\subsection{Cheaters or pollinators? Bee behaviour and bee-flower dimensions}

To describe bees' behaviour and visitation frequency throughout the day, we randomly assigned 30 previously labelled individuals, and conducted focal observations based on the protocol by Dafni et al. (2005). We performed observations throughout the daylight period, so that by the end of the flowering period we had $60 \mathrm{~h}$ of observation. During each interval of time, we recorded how many flowers were visited by each bee species per plant, the time the bees spent in each flower and the type of resource they collected. Later, we classified the bee visitors as pollinators or as different sorts of cheaters according to Inouye (1980). Additionally, we measured the thorax's diameter of the five specimens of each bee species and the tube diameter in the corolla region where the anthers and stigma are placed using 27 flowers from 9 plants (three flowers/plant) to describe the morphological adjustment between the bees' bodies and the floral tubes.

\subsection{Cheaters or pollinators? Bee efficiency in the production of viable seeds}

Considering that nectar-robbing bees never touch the flowers' reproductive structures, since they access nectar from the outside of the flower, we created two field experimental treatments to evaluate the role of bees that access floral resources from inside the flower, on seed formation: 'natural visitation' (natural seed set from all potential bee visitors) and 'small bees' (seed set excluding medium-sized bee visits). We also created one additional experiment: 'floral visitor exclusion' in order to check the need of a pollen transfer vector in the studied population.

The treatment in which the flowers were exposed to 'natural visitation' allowed all bee species to visit flowers of 10 different plants. These plants showed a mean of $3.1 \pm 1.9$ inflorescences and each inflorescence had $126.3 \pm 55.9$ flowers. In this treatment all the flowers produced by all the inflorescences of each plant were exposed to floral visitors during the entire reproductive period, which lasted around 90 days. In the 'small bees' treatment, we prevented the occurrence of medium and large-sized bees' visits by covering 14 inflorescences from 14 plants (one inflorescence/plant) with a wire mesh box with holes of $10 \mathrm{~mm}$ of diameter that only allowed the entrance of small bees. In this treatment all the flowers produced by the caged inflorescences ( $68.3 \pm 34.9$ flowers) were 
exposed to small bees throughout their entire reproductive period that lasted around 40 days. The 'floral visitor exclusion' experiment was performed in order to verify the potential seed set in the absence of a biotic vector. In the 'floral visitor exclusion' treatment, we maintained all the flowers, produced in five inflorescences from five plants (130.4 \pm 58.2 flowers/inflorescence/plant), isolated with bridal veil bags to avoid any visitation throughout the entire reproductive period, which lasted around 40 days.

Additionally, we performed a manipulative experiment using dead medium-sized bee specimens to describe their potential in pollen transferring. In this experiment, we simulated natural visits of medium-sized bees on 100 functional experimental flowers (previously bagged) from 10 plants (10 flowers/plant). We used specimens of two native species Bombus morio and Eulema nigrita, both species described as pollinators of J. caroba by Guimarães et al. (2008). For the experimental procedure, each bee specimen (rehydrated in a moist chamber) was glued to the tip of a popsicle stick. These bees were sequentially introduced in four flowers, with intact pollen loads (previously bagged), that acted as pollen donors (one flower/plant). The dorsal region of the bees' bodies was loaded with pollen grains from the four flowers. Subsequently, we used these bees to perform 100 simulated visits to the experimental flowers ( 50 visits with each bee species). We re-inserted the bee specimens into new pollen donor flowers after every five sequences of simulations of visits. Experimental flowers were maintained bagged until flower abscission or complete fruit development.

By the end of the reproductive season, we evaluated the inflorescences and collected the fruits from all experiments; we counted the number of seeds per fruit and conducted laboratory germination tests. The seeds were deposited in Petri dishes containing paper towel moistened with distilled water and were kept in a germination chamber at $25^{\circ} \mathrm{C}$ with a 12 -h photoperiod. Seed germination was evaluated daily for 20 days. The seeds were considered viable when the primary root protruded within this period (Brasil, 2009). Finally, the number of seeds per fruit and the proportion of seeds germinated were compared among the 'natural visitation' and 'small bees' treatments. The comparison between the results of these two treatments, which included similar stochasticity associated with natural visitation, allowed us to assess the contribution that small and medium-sized bees make to the production of viable seeds.

\subsection{Visitation pattern throughout the day}

To describe the number of $J$. caroba flowers visited per each bee species throughout the day, we randomly monitored 30 previously labelled plants. Every hour, from 7:00 to $18: 00 \mathrm{~h}$, we recorded how many flowers per plant were visited by each bee species.

\subsection{Cheater's effect on pollen and nectar removal}

To test the effect of small bees on pollen removal from the anthers, we used an exclusion experiment in which some inflorescences were bagged with bridal veil bags to avoid all visitors ('exclusion of floral visitors' treatment), and others were covered with wire mesh boxes (as in item 2.3.) excluding medium- and large-sized bees and allowing only small bees' visits ('small bees' treatment). We applied both treatments on two inflorescences in each plant ( $n=5$ plants), and after three days, we collected the four anthers from three flowers per inflorescence from both treatments for pollen grain quantification.

We tested the nectar removal effect using a day-long natural experiment with three treatments. Since, in this population, there was a large incidence of floral damage (in slit format) at the base of the corolla tube caused by the primary nectar robber Oxaea flavescens (personal observations), we included one treatment to investigate the effect of Oxaea flavescens in the nectar depletion of J. caroba flowers. The three treatments applied were: (i) all floral visitors excluded from the flowers (bagged flowers - control treatment); (ii) flowers exposed to all potential floral visitors except Oxaea flavescens - flowers that remained without any slit throughout the day; and (iii) flowers exposed to all potential floral visitors including Oxaea flavescens - flowers with at least one slit recorded in the corolla base early in the morning (at the first nectar volume measurement). In this experiment, we used first-day flowers, distributed on 17 plants and totalling 104 flowers. We evaluated each treatment at three different times, 8:00 h, 13:00 h and 18:00 h. We sampled one flower per treatment per time in each plant. We collected the flowers from the field and individually wrapped them to transport to the laboratory, where we performed the nectar volume measurements using capillary tubes to withdraw the nectar from each one of them.

\subsection{Testing the effect of floral damage caused by nectar robbers on pollen transfer}

Since the slits on the floral tube's base, caused by the primary robber Oxaea flavescens, can negatively affect pollination due to visual or chemical changes in J. caroba flowers, we tested the effect of slits on the number of pollen grains deposited on the receptive stigma surface. For this purpose, we used a natural experiment to compare pollen deposition on flowers exposed to bee visitation: (i) without any slits caused by Oxaea flavescens (control flowers); and (ii) with at least one slit caused by Oxaea flavescens present at the corolla base since early in the morning, before any potential pollinator visit (damaged flowers). We randomly assigned 20 plants in the population and marked all opening flowers available per plant between 7:00 h and 8:00 h. At 18:00 h, we collected all the flowers from both treatments, considering as 'control flowers' only the flowers that remained intact, without any slits caused by Oxaea flavescens, until the end of the experiment. We collected each flower's pistil and fixed them in FPA solution. Then, in the laboratory, we counted and compared the amount of pollen grains on the stigmatic surface of flowers with and without Oxaea flavescens slits.

\subsection{Statistical analysis}

Bee visitation pattern throughout the day was modelled separately for each ecological group of bees (pollinators, nectar robbers, nectar thieves and pollen thieves) and the three most frequent floral visitors (Bombus morio, Oxaea flavescens and Ceratina sp.). Given that we did not have any a priori reason to choose a particular parametric shape to describe the relationship between bee visitation (per ecological groups or bee species) and daytime, we applied generalized additive models (GAM) which modeled daytime as non-parametric smoothed function $s$ to explain the number of flowers visited per hour (counting variable). Zero inflated Poisson error distribution was used in each GAM model. We also modelled the probability of occurrence of nectar robbery damages (binary variable) throughout the day using a generalized linear mixed model (GLMM) with binomial error distribution, considering daytime as a continuous fixed factor and the number of flowers nested in individual plants as a random variable. We compared the number of seeds per fruit (integer variable) and the proportion of germinated seeds (continuous variable), among the three treatments, using generalized linear models (GLM) with Poisson and Binomial error distribution, respectively. Subsequently, we applied Tukey's honestly significant difference (HSD) post hoc analysis to compare all possible pairs of means. We compared the number of removed pollen grains (counting variable) between 'control' and 'small bee' treatments in a generalized linear mixed model (GLMM) with Poisson error distribution and individual plants as a random variable. 

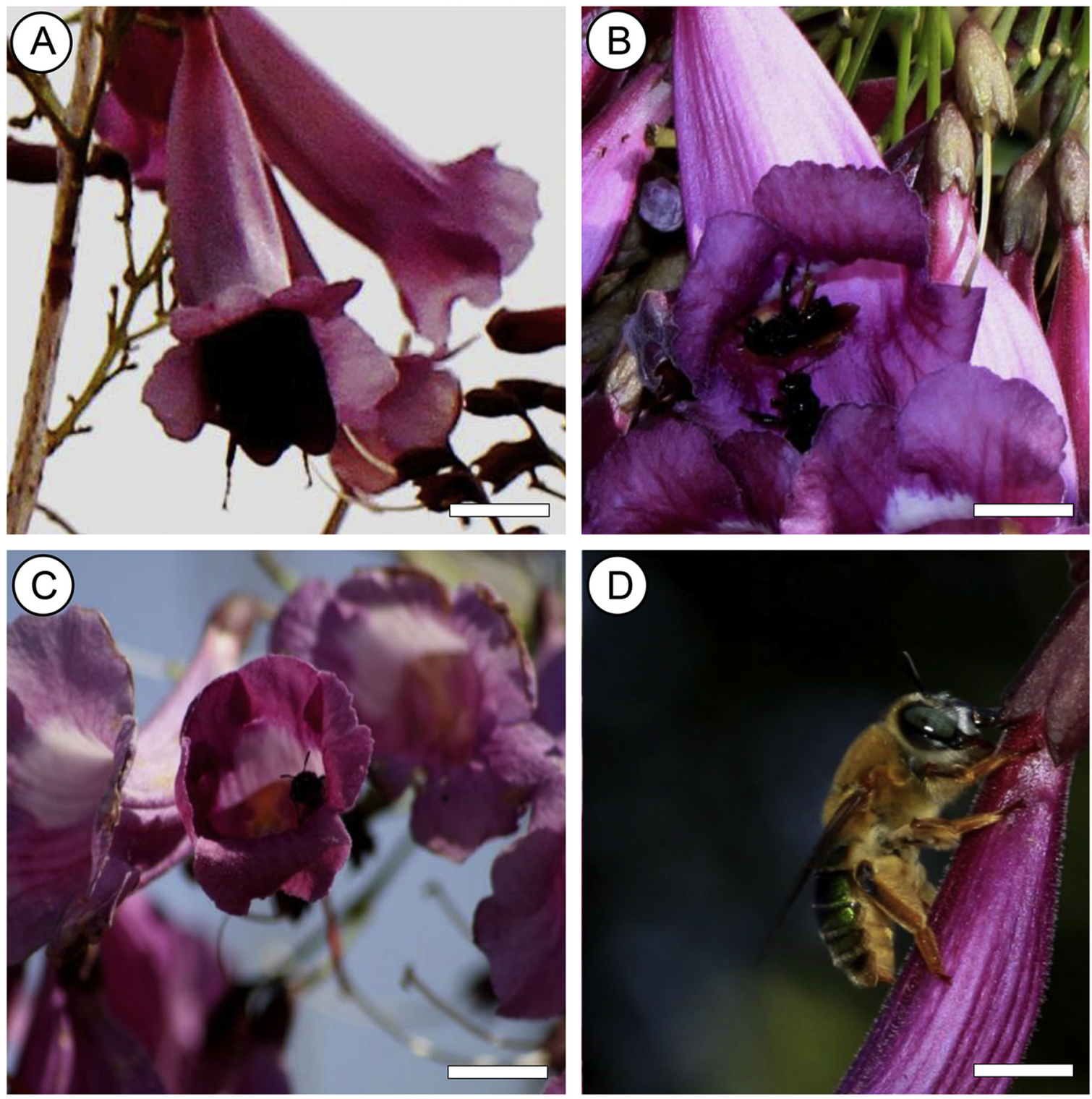

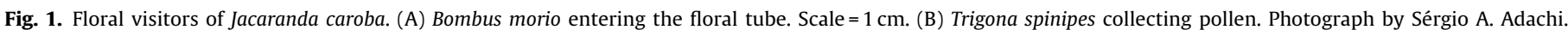

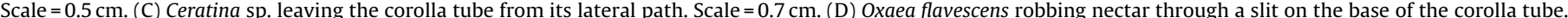
Photograph by Sérgio A. Adachi. Scale $=0.5 \mathrm{~cm}$.

We compared the nectar volume variation (continuous variable) throughout the day among the three treatments using a general linear mixed model (LMM) with normal error distribution and individual plants as a random variable. We applied HSD post hoc analysis in order to compare all possible pairs of means. We compared the number of pollen grains (integer variable) deposited on the stigmatic surface of each flower between the two treatments using a GLMM with Poisson error distribution and individual plants as a random variable. All statistical analysis were carried out in $R$ v. 3.1.1 (R Development Core Team, 2016) with standard and additional packages: mgcv (Wood, 2001), plotrix (Lemon, 2006) and glmm ADMB (Bolker et al., 2012).

\section{Results}

\subsection{Cheaters or pollinators? Bee behaviour and bee-flower dimensions}

We observed visits from medium- and small-sized bees belonging to different functional groups in Jacaranda caroba flowers. These bees were classified as pollinators, pollen thieves, nectar thieves or nectar robbers, based on their behaviour during flower foraging. Potential pollinators: Bombus morio is a medium-sized bee species, with a thorax diameter of $10.07 \mathrm{~mm} \pm 0.84$, that entered the floral tube facing the nectar chamber (Fig. 1A), touching the flower's reproductive structures due to the compatibility between its body size and the diameter and length of $J$. caroba floral tube. This bee species visited on average two flowers per plant, expending four seconds per visit (Table 1). Potential pollen thieves: Plebeia cf. droryana and Trigona spinipes (Fig. 1B) are small bees, with thorax diameters of $1.6 \mathrm{~mm} \pm 0.2$ and $2.5 \mathrm{~mm} \pm 0.3$, respectively. They landed on the anthers and actively collected pollen grains, usually without contacting the stigmatic surface. While Plebeia cf. droryana visited one flower per plant, for about $20 \mathrm{~s}$, Trigona spinipes bees also visited one flower per plant, but for about $40 \mathrm{~s}$ (Table 1). Potential nectar thieves: Ceratina sp. (Fig. 1C) and Epicharis sp. are small bees, with thorax diameters of $2.7 \mathrm{~mm} \pm 0.2$ and $4.9 \mathrm{~mm} \pm 0.5$, respectively. They apparently searched for nectar at the base of the floral tube. Both species usually entered through the lateral portions of 
Table 1

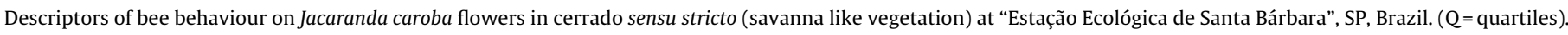

\begin{tabular}{|c|c|c|c|c|c|c|c|c|c|}
\hline \multirow[t]{2}{*}{ Floral visitors } & \multirow[t]{2}{*}{$\mathrm{N}$} & \multicolumn{3}{|c|}{ Number of visits within the same plant } & \multicolumn{3}{|c|}{ Time spent per visit per flower (seconds) } & \multicolumn{2}{|c|}{$\begin{array}{l}\text { Contact with reproductive } \\
\text { structures }\end{array}$} \\
\hline & & Media (Mín, Máx) & $1 \mathrm{Q}$ & $3 Q$ & Median (Mín, Máx) & $1 \mathrm{Q}$ & $3 Q$ & Anthers & Stigma \\
\hline Bombus morio & 5 & $2(1,4)$ & 2 & 3 & $4(2,7)$ & 4 & 4 & yes & yes \\
\hline Trigona spinipes & 8 & $1(1,5)$ & 1 & 2.5 & $38(2,390)$ & 6 & 51 & yes & eventually \\
\hline Plebeia cf. droryana & 10 & $1(1,4)$ & 1 & 1 & $21(4,129)$ & 9 & 31.5 & yes & eventually \\
\hline Ceratina sp. & 43 & $3(1,10)$ & 1.5 & 4 & $11.5(1,149)$ & 5 & 23.7 & eventually & eventually \\
\hline Epicharis sp. & 5 & $1(1,3)$ & 1 & 1 & $6(3,20)$ & 3.5 & 7.5 & eventually & eventually \\
\hline Oxaea flavescens & 25 & $4(1,8)$ & 2 & 4 & $3(1,10)$ & 2 & 4 & No & No \\
\hline
\end{tabular}

the corolla tube (Fig. 1C), facilitating their access to the nectar chamber on the tube's base. These bees did not touch the reproductive structures due to their reduced body sizes as compared to the flower tube dimensions and to their behaviour. On average, Ceratina sp. stayed inside the floral tube for $12 \mathrm{~s}$ and visited three flowers per plant (Table 1), while Epicharis sp. bees remained inside the floral tube for around $6 \mathrm{~s}$ and visited only one flower per plant (Table 1 ). Nectar robbers: Oxaea flavescens is a medium-sized bee, with a thorax diameter of $6.4 \mathrm{~mm} \pm 0.4$ that robbed nectar through a slit made, from the outside, on the base of the corolla tube (Fig. 1D). These bees exhibited an evident aggressive behaviour, flying around the flower towards other visitors. On average, Oxaea flavescens stayed on the flowers for three seconds feeding on nectar, and visited four flowers per plant (Table 1).Jacaranda caroba flowers showed a diameter of $10.0 \mathrm{~mm} \pm 1.0$ in the compressed region of the corolla tube.

\subsection{Cheaters or pollinators? Bee efficiency in the production of viable seeds}

We detected significant differences in seed production among the three treatments $\left(F=46.7 ; \mathrm{p}<0.001, \mathrm{~N}_{\text {plants }}=29\right)$. We observed a significantly higher average number of seeds per fruit in the inflorescences exposed to all visitors ('natural visitation' treatment) when compared to the inflorescences visited only by small bees ('small bee' treatment) or the inflorescences that were excluded of all visitors ('floral visitor exclusion' treatment) (Fig. 2A). Flowers visited only by small bees produced $19.3 \pm 12.0$ (mean \pm SD) seeds per fruit, while flowers exposed to all visitors produced $45.3 \pm 19.7$ seeds per fruit. Considering the proportion of germinated seeds, we observed a similar pattern among treatments $(\mathrm{F}=750.6, \mathrm{p}<0.001$, $\mathrm{N}_{\text {plants }}=24$ ) in which the 'natural visitation' treatment had around $65 \%$ of seed germination, and the 'small-bee' treatment had no seeds germinating (Fig. 2B). In this second analysis, we did not include the 'floral visitor exclusion' treatment because those plants did not produce any seeds for the germination test.

Additionally, flowers used in the 'medium-sized bee' manipulative experiments produced on average $60.4 \pm 33.0$ seeds with $66 \%$ of seed germination.

\subsection{Visitation pattern throughout the day}

Bombus morio was the only species classified as pollinator, while Ceratina sp. (nectar thief) and Oxaea flavescens (nectar robber) were the most frequent cheaters registered on the sampled flowers (Fig. 3A). The bee visitation pattern throughout the day on J. caroba flowers varied among ecological groups and bee species (Fig. 3B-C). Ceratina sp. and pollen thieves' activity had a small overlap with pollinators' in the early morning (before 9:00 h), but both cheaters showed visit peaks later, with an increase in the number of visited flowers from 9:00 h on (Fig. 3B-C). Among bee species, we also modelled the visitation pattern of the potential pollinator (Bombus morio) and of the most frequent nectar cheaters (Ceratina sp. and Oxaea flavescens). Bombus morio had its visits peak between

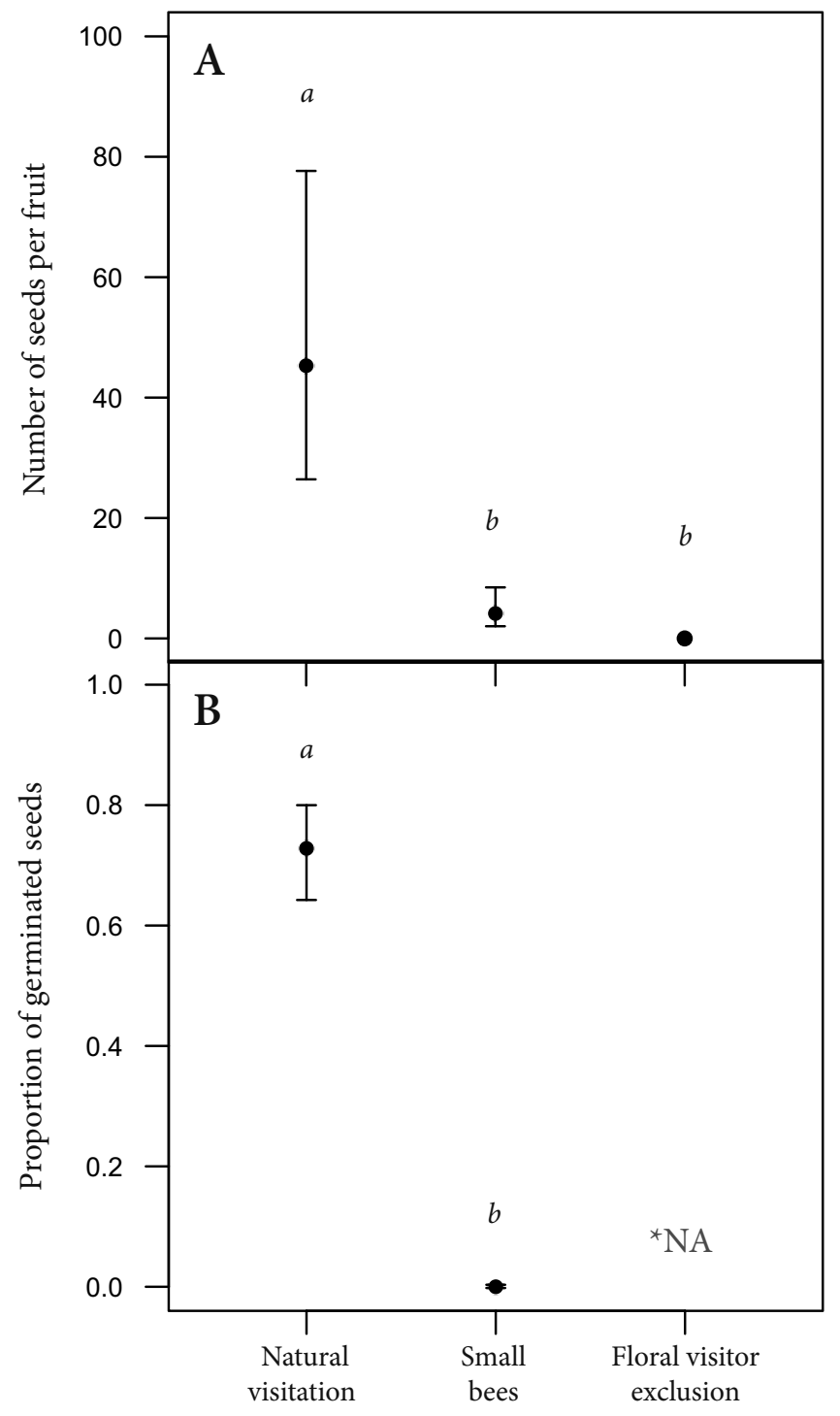

Fig. 2. Bee efficiency as Jacaranda caroba pollinators. (A) Average number of seeds per fruit. (B) Proportion of germinated seeds. Different letters indicate significant differences between treatments $(\mathrm{p}<0.05)$. The means and standard errors are backtransformed from the log-scale. ${ }^{*} \mathrm{NA}=$ Treatment not included in the analysis due to the absence of seeds.

8:00 $\mathrm{h}$ and 9:00 $\mathrm{h}$ in the morning, overlapping the period of Ceratina sp. visits (Fig. 3C). In particular, Ceratina sp. bees fed on nectar throughout the day, being the most common consumer of J. caroba nectar. Oxaea flavescens started its visits around $8: 30 \mathrm{~h}$ with a gradual increase of its visitation rate and an overlap with Bombus morio, showing its peak of visits around 10:30 h. A detailed statistical out- 

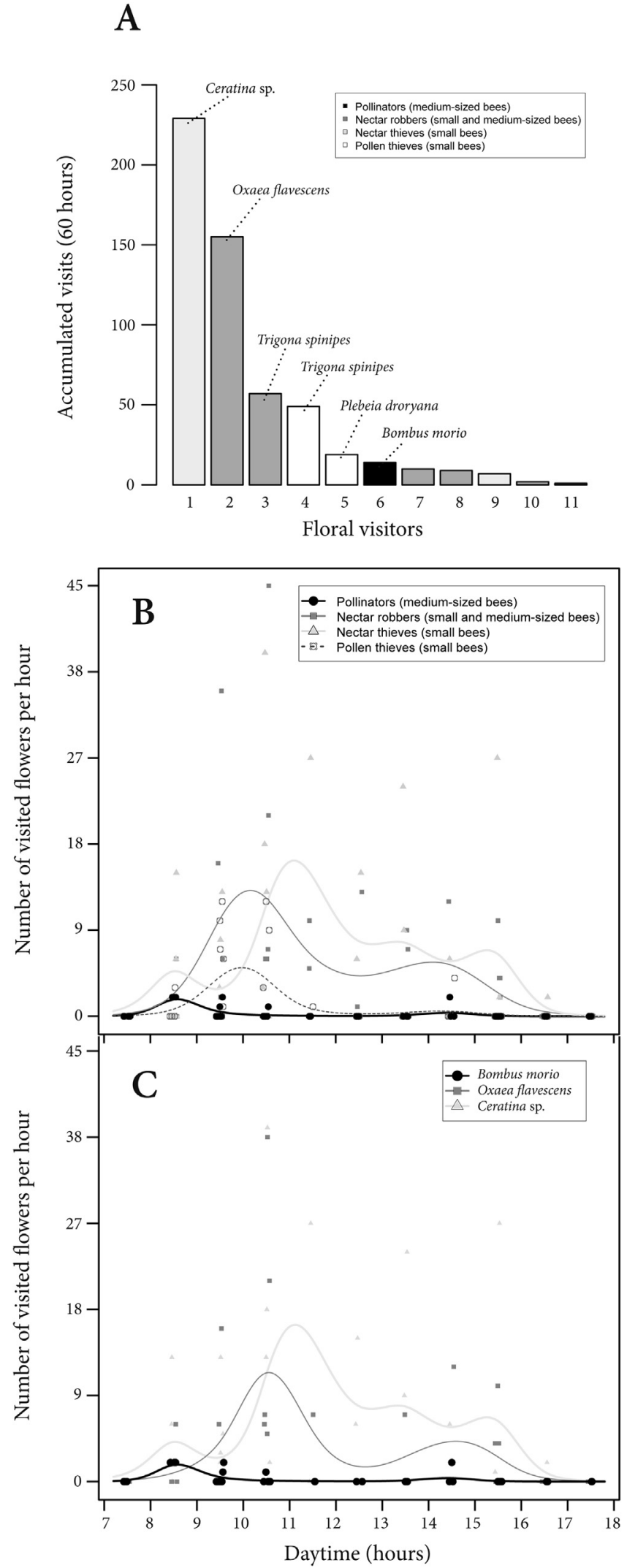

Fig. 3. Bees visits to Jacaranda caroba flowers. (A) Accumulated number of visited flowers for every bee species registered during observations. (B) Number of flowers visited per hour by each of the following ecological groups: pollinators, nectar robbers, nectar thieves and pollen thieves. (C) Number of flowers visited per hour by the pollinator, Bombus morio, and by the most frequent cheaters, Oxaea flavescens and Ceratina sp.

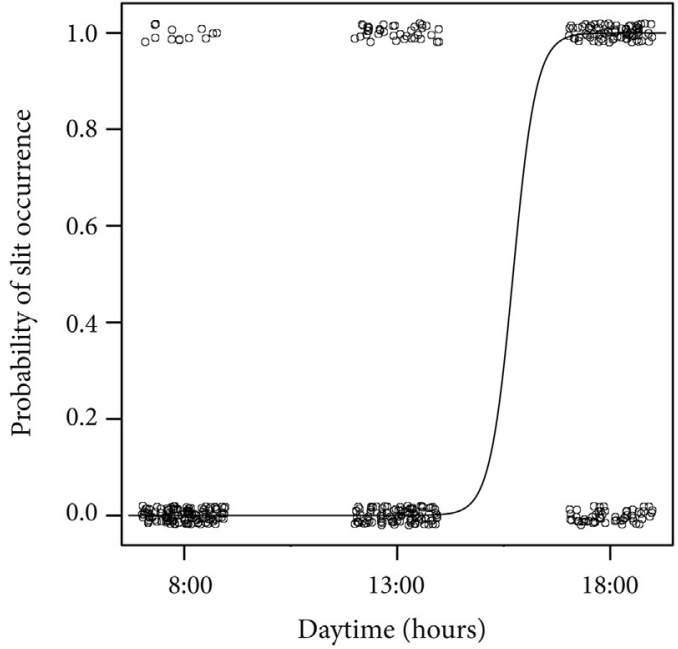

Fig. 4. Probability of floral damages (slits) occurrence caused by Oxaea flavescens throughout the day in Jacaranda caroba flowers.

put from the modelling of bee visitation pattern throughout the day can be found in Supplementary material 1 .

Nectar robbery damages on first-day flowers increased throughout the day and were represented exclusively by Oxaea flavescens slits. The probability of Oxaea flavescens slits occurrence increased considerably after $13: 00 \mathrm{~h}(\mathrm{Z}=7.95, \mathrm{p}<0.01$, $\mathrm{N}_{\text {flowers }}=133, \mathrm{~N}_{\text {plants }}=20$ ) (Fig. 4). The number of observed slits was little at $8: 00 \mathrm{~h}$ (9.77\% of damaged flowers in $40 \%$ of plants) and increased until 13:00 h (27.82\% of damaged flowers in 75\% plants). In the afternoon (between $13: 00 \mathrm{~h}$ and $18: 00 \mathrm{~h}$ ), the number of flowers with Oxaea flavescens slits duplicated (57.89\% of damaged flowers) and slits were present in almost all of the sampled plants (90\% of plants).

\subsection{Cheater's effect on pollen and nectar removals}

We detected a reduction in the amount of pollen grains on the anthers of the flowers exposed to small bees' visits, when compared with unvisited flowers ('floral visitor exclusion' - control) $(Z=8.38$, $\left.\mathrm{p}<0.01, \mathrm{~N}_{\text {flowers }}=15, \mathrm{~N}_{\text {plants }}=5\right)$. After three days of exposure, pollen thieves removed about $54 \%$ of the pollen grains produced per flower. On average, each J. caroba flower (4 anthers) produced $28850.0 \pm 7216.5$ (mean \pm SD) pollen grains, and remained with $13160.0 \pm 6626.3$ after natural pollen removal by small bees.

We found no significant difference in nectar volume throughout the day $\left(F=0.41, p=0.52, N_{\text {flowers }}=104, N_{\text {plants }}=17\right)$. Nevertheless, nectar volume varied significantly between treatments $(F=6.28$, $\mathrm{p}<0.01$ ). Bagged flowers (control) had on average once to twice more nectar content (1.5-3.0 $\mu \mathrm{l}$ more nectar) than flowers exposed to visitors (with or without Oxaea flavescens slits) in the early morning (Fig. 5). Flowers exposed to visitors with or without slits had the same pattern of nectar volume throughout the day, showing a higher number of flowers without any nectar after midday (Fig. 5).

\subsection{Effect of floral damage caused by nectar robbers on pollen transfer}

Among the 133 first-day flowers evaluated in this experiment, only $9.7 \%$ had Oxaea flavescens slits in the early morning (7:00 h). By the end of the day (18:00 h), $57.9 \%$ of the flowers had damages (slits) caused by nectar robbers. We didn't detect significant differences in the number of pollen grains deposited on the stigma of flowers with and without slits $\left(\mathrm{Z}=1.57, \mathrm{p}=0.12, \mathrm{~N}_{\text {flowers }}=68, \mathrm{~N}_{\text {plants }}=19\right)$ (Fig. 6). 


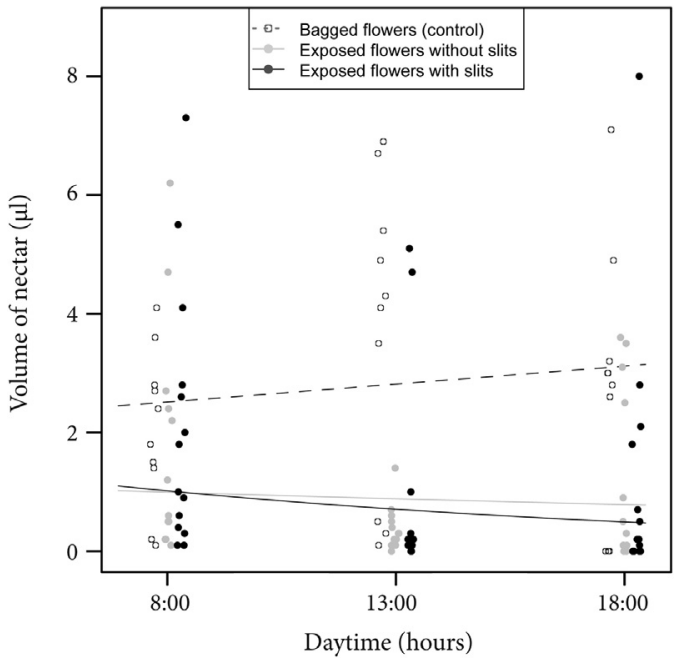

Fig. 5. Nectar volume in Jacaranda caroba flowers in three treatments: bagged flowers (flowers excluded from all floral visitors); exposed flowers without slits (flowers exposed to all potential floral visitors except Oxaea flavescens) and exposed flowers with at least one slit (flowers exposed to 0 . flavescens and all the other potential floral visitors).

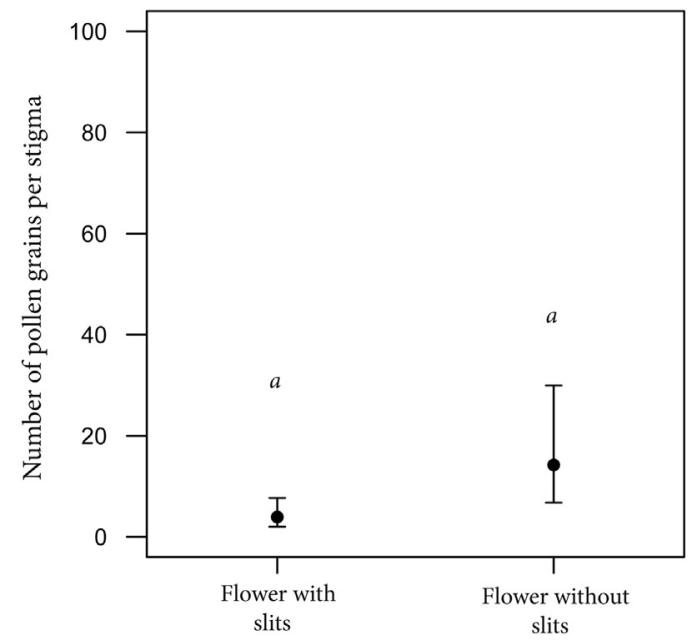

Fig. 6. Number of pollen grains deposited on the stigmas of Jacaranda caroba flowers with and without Oxaea flavescens slits. Different letters indicate significant differences between treatments $(\mathrm{p}<0.05)$. The means and standard errors are backtransformed from the log-scale.

\section{Discussion}

In this study we experimentally evaluated the role of bee species as pollinators or cheaters and detailed the temporal pattern of bee visitation throughout the day. Once we had unequivocally stated which group of bee species acted as cheaters or as pollinators, we explored the potential effect of cheaters on the depletion of floral resources, including nectar and pollen, and ultimately we investigated the potential effect of damages caused by nectar robbers on pollen transfer.

Jacaranda caroba flowers received visits from different bee species, each one showing a distinct ecological strategy of resource exploitation. Cheaters in our system exhibited higher richness, abundance and number of flowers visited per hour. Since Darwin (1841), cheaters have been described as widely associated to plantpollinator mutualisms. In fact, the scope of this statement was confirmed in our study, since $98 \%$ of flowers were accessed by visitors that exploited resources without accomplishing pollen transfer.
The approach used here to evaluate the outcome of bee visits to J. caroba flowers allowed a high accuracy in differentiating pollinators and cheaters. We determined the efficiency in pollen transfer by each ecological group based on Ne'eman et al. (2010), who consider that pollen transfer is efficient when the pollen deposited by pollinators is suitable to ensure plant reproduction, in terms of quality and quantity. We used viable seed production as an indicator of reproductive success, which would be a consequence of pollen deposition efficiency. In our study, in experimental simulated visits, medium-sized bees (Bombus morio and Eulema nigrita) originated a high amount of viable seeds, similar to the amount produced in natural conditions, indicating that these bees are in fact efficient pollinators of $J$. caroba. These bee species were also considered efficient pollinators of $J$. caroba based on their behaviour and dimensions by Yanagizawa and Maimoni-Rodella (2007) and Guimarães et al. (2008). In our study system Bombus morio was the only species recorded legitimately visiting the flowers of $J$. caroba and having a body size compatible with the floral tube dimensions.

Small bees that collect floral resources in Jacaranda species can occasionally touch the stigma surface (Maués et al., 2008; MiletPinheiro and Schlindwein, 2009). Thieves that contribute to pollen deposition on the stigma were also described for other plant species as Lapageria rosea (Valdivia and González-Gómez, 2006) and Aloe maculata (Duffy and Johnson, 2011). However, not all the pollen grains deposited onto the stigma's surface will lead to seed production, especially if small bees mainly transfer self-pollen during the pollen collection manoeuvres. In fact, our results showed that, even though small bees acted as pollen transfer vector, they had no relevance for $J$. caroba sexual reproduction and could be generically treated as cheaters. The lack of viable seeds from small-bees interactions with flowers occurs because $J$. caroba is predominantly xenogamous (Yanagizawa and Maimoni-Rodella, 2007; Guimarães et al., 2008) and most of the plants are not expected to self-fertilize.

The production of seeds from small bees' visits to J. caroba flowers was most probably due to the activity of pollen thieves that directly contacted the anthers during pollen collection. Indeed, Ballantyne et al. (2015) showed that pollen thieves were more actively in contact with the anthers and, thus, deposited greater amounts of pollen on the stigma's surface than nectar thieves. Additionally, nectar thieves entered $J$. caroba flowers via the lateral portion of the corolla tube to access the nectar chamber, without entering the corolla central region, where the reproductive structures are located. Besides, the thorax dimensions of Ceratina sp. and Epicharis sp. do not match the floral tube dimensions to guarantee the contact with the reproductive structures during the resource collection. In conclusion, small bees acted only as cheaters without contributing to seed production, regardless of being nectar or pollen thieves.

We registered different visitation patterns for cheaters and pollinators throughout the day. Pollinators showed a slight peak in the early morning, while the most frequent cheaters, Ceratina sp. (nectar thief) and Oxaea flavescens (nectar robber), fed on flowers all day long, increasing visitation during mid-morning. Although Ceratina sp. showed a slight peak concomitant with pollinators in the early morning, the nectar content available in this period is higher than in the mid-morning due to the presence of recently opened first-day flowers full of nectar. So, the activity of nectar thieves probably does not have such a strong impact on the food supply to pollinators.

Temporal structure has been suggested as an important process influencing the outcome of cooperation (see references in Barker and Bronstein, 2016). When cheaters exploiting resources are active before mutualists, the costs to the plant is higher, but if mutualists' visits occur first, as in our studied system, this cost is diminished (Barker and Bronstein, 2016). Thus, the cost for the Jacaranda-bees system is probably low, as pollinators' visits and 
pollen transfer happened before cheaters' visits (pollen and nectar), with only a slight overlap with Ceratina sp. visits.

In a scenario where plants establish interactions with different groups of pollinators and robbers in the community, nectar robbers may exploit specific plant species due to competition for resources (Rojas-Nossa et al., 2016). Competition is an expected scenario when resources are reduced and the abundance of exploiters is high (Ricklefs, 2010). Some theoretical models have been proposed by Wang et al., (2012) and Wang (2013) to explain the persistence of pollination mutualisms in the presence of nectar robbers (Bronstein et al., 2006; Irwin et al., 2010) in real situations. Robbers' needs may be determinant to pollinators' persistence, so if plants provide enough resource for the robbers' maintenance, they can always invade the plant-pollinator system. On the other hand, pollinators can persist if the robbers' efficiency is relatively lower than pollinators' (Wang, 2013). In the Jacaranda-bees system, pollinators' visits in the early morning could represent a higher efficiency in the use of resources, as compared to the main robbers', Oxaea flavescens. Additionally, from the mid-morning on, all cheaters compete for nectar, which may represent a relatively low efficiency of these ecological groups in the use of resources as compared to pollinators. So, actually, competition between mutualists and cheaters does not seem to be occurring in this system at the present moment. However, it could have shaped the observed temporal sequence of visits if, for example, in a past moment, robbers and thieves invaded the plant-mutualist system leading to a displacement of the period of pollinator visits due to the competition.

Besides, the clustered pattern of pollinators' visits in the early morning could also be triggered by the agonistic behaviour displayed by some cheaters. In fact, we observed Oxaea flavescens, a specialized bee species that steals nectar from the flowers of several Bignoniaceae species (Gottsberger and Silberbauer-Gottsberger, 2006), showing aggressive behaviour in relation to other floral visitors that were flying around $J$. caroba flowers. Here, the increase of Oxaea flavescens visitation in the morning coincides with the decrease of Bombus morio visitation. Considering this, Oxaea flavescens could exert an ecological pressure on pollinators that has moved their visitation period to the early morning, a hypothesis that can be further explored in this system. Alternatively, in the study area, other nectar sources may become available later in the morning for medium-sized bees, so they visit J. caroba flowers early in the morning and move on to forage in other species with higher nutritional offer, for example.

Pollen removal by thieves or robbers may lead to pollen limitation in the system by depleting male gametes (Carmo et al., 2004; Hargreaves, 2007) and reducing cross-pollination rates (Hargreaves et al., 2009). In J. caroba the main pollen thieves were Trigona spinipes and Plebeia cf. droryana that removed about $54 \%$ of the pollen grains available in each flower. Hargreaves et al. (2010) showed even more intense pollen theft in other natural systems. Besides, considering that the pollen: ovule ratio represents the relative number of male and female gametes (Michalski and Durka, 2009), pollen thieves may practically halve the male function of a $\mathrm{J}$. caroba flower, negatively affecting pollination. Although pollen thieves' visits occur after pollinators' visits, in our system second and third day flowers may be more strongly affected by pollen removal, and consequently they may act mainly as functionally female flowers.

The cheaters' effect on nectar removal may affect the plants' reproductive success in different ways (Maloof and Inouye, 2000; Irwin et al., 2010). Considering that, in nature, a high percentage of flowers are robbed, it is important to determine the impact of nectar robbers and their variations on flowers (Maloof, 2001; Wang, 2013; Barker and Bronstein, 2016). In J. caroba, the depletion of nectar can be mainly due to the visits of cheaters, which were more frequent and more widely distributed throughout the day. Indeed, any exploiter could easily withdraw the limited amount of nectar available in J. caroba flowers, which may constrain the exploitation by robbers and thieves. So, this low energy supply may allow pollinators and nectar cheaters to coexist, in a density-dependent system similar to the one proposed by Wang (2013), since plants with abundant nectar are more subjected to being robbed (Rojas-Nossa et al., 2016).

In $J$. caroba, we did not detect significant differences in the amount of pollen deposited on the stigmas in flowers with and without damages caused by nectar robbers. This could be an indicative that robbery damage does not influence pollinators' choice of flowers in our system, similar to the verified by Richardson (2004) in another system. On the other hand, the absence of difference in pollen transfer between robbed and unrobed flowers of $J$. caroba may be because such transference occurs mainly prior to robbing.

\section{Conclusions}

In conclusion, only medium-sized bees were considered pollinators of J. caroba, while small bees acted as cheaters. Pollen thieves, in three days, halved the amount of male gametes available for pollination, while nectar thieves and robbers withdrew approximately $70 \%$ of the nectar volume during their peak of visits. It seems that, in the Jacaranda-bees system, the removal of nectar affects pollinator activity more than floral damage.

This scenario suggests a competition for nectar among these distinct ecological groups of bees. However, when pollinators visit $J$. caroba flowers in the early morning, there might be a smaller competition pressure among visitors due to the greater amount of resources available, especially in recently opened flowers, and due to the low frequency of cheaters visits. So, we hypothesize that competition in this system may occur mainly among the antagonists (nectar robbers and thieves) after the early morning, since their peaks of visits are overlapped. The persistence of the pollination mutualism may be due to a temporal escape of pollinators from the "rush hour" period, when competition is tougher and food resources are scarcer.

\section{Acknowledgements}

Funding: This work was supported by 'Fundação de Amparo à Pesquisa do Estado de São Paulo, FAPESP' (Biota Program Proc. 2008/55434-7 and PD-Fellowship granted to E. Guimarães, Proc. 2009/17611-7) and 'Conselho Nacional de Desenvolvimento Científico e Tecnológico, CNPq' (Edital Universal Proc. 446949/2014-0). We thank the 'Estação Ecológica de Santa Bárbara' and 'Instituto Florestal do Estado de São Paulo' for area infrastructure, Sérgio A. Adachi for the photographic registers, Elaine Françoso for the identification of Bombus morio, Alexandre Zuntini for the identification of the plant species, Priscila T. Tunes for the English review, and the students from the laboratory of 'Ecology and Evolution of Plant-Animal Interactions' for field support.

\section{Appendix A. Supplementary data}

Supplementary data associated with this article can be found, in the online version, at http://dx.doi.org/10.1016/j.flora.2016.08. 005.

\section{References}

Ballantyne, G., Baldock, K.C., Willmer, P.G., 2015. Constructing more informative plant-pollinator networks: visitation and pollen deposition networks in a heathland plant community. R. Soc. 282, 1-9.

Barker, J.L., Bronstein, J.L., 2016. Temporal structure in cooperative interactions: what does the timing of exploitation tell us about its cost? PLoS Biol. 14, e1002371. 
Bolker, B., Skaug, H., Magnusson, A., Nielsen, A., 2012. Getting Started with the glmmADMB Package.

Brasil, 2009. Regras para análise de sementes. Ministério da agricultura, pecuária e abastecimento. Secretaria de defesa agropecuária, Brasília.

Bronstein, J.L., 1994. Our current understanding of mutualism. Q. Rev. Biol. 13, 315-347.

Bronstein, J.L., Alarcón, R., Geber, M., 2006. The evolution of plant-insect mutualisms. New Phytol. 172, 412-428.

Carmo, R.M., Franceschinelli, E.V., Silveira, F.A., 2004. Introduced honeybees (Apis mellifera) reduce pollination success without affecting the floral resource taken by native pollinators. Biotropica 36, 371-376.

Castro, S., Loureiro, J., Ferrero, V., Silveira, P., Navarro, L., 2013. So many visitors and so few pollinators: variation in insect frequency and effectiveness governs the reproductive success of an endemic milkwort. Plant Ecol. 214, 1233-1245.

Chittka, L., Thomson, J.D., 2001. Cognitive Ecology of Pollination Animal Behavior and Floral Evolution. Cambridge, University Press, U.K.

Dafni, A., Kevan, P.G., Husband, B.C., 2005. Practical Pollination Biology. Enrivoquest, Ltd., Cambridge, Ontario, Canada (590p).

Darwin, C., 1841. Humble-Bees. In: Barret, P.H. (Ed.), The Collected Papers of Charles Darwin. University of Chicago, Illinois 1, pp. 142-145.

Duffy, K.J., Johnson, S.D., 2011. Effects of pollen reward removal on fecundity in a self-incompatible hermaphrodite plant. Plant Biol. 13, 556-560.

Genini, J., Morellato, L.P.C., Guimarães, P.R., Olesen, J.M., 2010. Cheaters in mutualism networks. Biol. Lett. 6, 494-497.

Gentry, A.H., Morawetz, W., 1992. Bignoniaceae tribe tecomeae. Flora Neotropica Monographs 25, 51-105.

Gottsberger, G., Silberbauer-Gottsberger, I., 2006. Life in the Cerrado - Pollination and Seed Dispersal. Reta Verlag, Germany.

Guimarães, E., Di Stasi, L.C., Maimoni-Rodella, R.D.C.S., 2008. Pollination biology of Jacaranda oxyphylla with an emphasis on staminode function. Ann. Bot. 102, 699-711.

Hargreaves, A.L., Harder, L.D., Johnson, S.D., 2009. Consumptive emasculation: the ecological and evolutionary consequences of pollen theft. Biol. Rev. 84, 259-276.

Hargreaves, A.L., Harder, L.D., Johnson, S.D., 2010. Native pollen thieves reduce the reproductive success of a hermaphroditic plant, Aloe maculata. Ecology 91, 1693-1703.

Hargreaves, A.L., 2007. The Ecological Effects of Pollen-stealing Insects on Plant Reproductive Success, M.Sc. University of Calgary.

Inouye, D.W., 1980. The terminology of floral larceny. Ecology 61, 1251-1253.

Irwin, R.E., Bronstein, J.L., Manson, J.S., Richardson, L., 2010. Nectar robbing: ecological and evolutionary perspectives. Ann. Rev. Ecol. Evol. Syst. 41, e292.

Lemon, J., 2006. Plotrix: a package in the red light district of R. R News 6, 8-12.

Maloof, J.E., Inouye, D.W., 2000. Are nectar robbers cheaters or mutualists? Ecology 81, 2651-2661.

Maloof, J.E., 2001. The effects of a bumble bee nectar robber on plant reproductive success and pollinator behavior. Am. J. Bot. 88, 1960-1965.
Maués, M.M., Oliveira, P.E.A.M., Kanashiro, M., 2008. Pollination biology in Jacaranda copaia (Aubl.) D. Don. (Bignoniaceae) at the Floresta Nacional do Tapajós Central Amazon, Brazil. Revista Brasileira de Botânica 31, 517-527.

Melo, A.C.G., Durigan, G., 2011. Plano de Manejo da Estação Ecológica de Santa Bárbara. Instituto Florestal/SEMA, São Paulo.

Michalski, S.G., Durka, W., 2009. Pollination mode and life form strongly affect the relation between mating system and pollen to ovule ratios. New Phytol. 183, 470-479.

Milet-Pinheiro, P., Schlindwein, C., 2009. Pollination in Jacaranda rugosa (Bignoniaceae): Euglossine pollinators, nectar robbers and low fruit set. Plant Biol. 11, 131-141.

Ne'eman, G., Jürgens, A., Newstrom-Lloyd, L., Potts, S.G., Dafni, A., 2010. A framework for comparing pollinator performance: effectiveness and efficiency. Biol. Rev. 85, 435-451.

R Development Core Team, 2016. 'R: A Language and Environment for Statistical Computing.'. R Foundation for Statistical Computing, Vienna, Austria, ISBN 3-900051-07-0, Available at http://www.R-project.org (accessed 30.07.16)

Richardson, S.C., 2004. Are nectar-robbers mutualists or antagonists? Oecologia $139,246-254$.

Ricklefs, R.E., 2010. A economia da natureza, sixth ed. Guanabara Koogan, Rio de Janeiro.

Rojas-Nossa, S.V., Sánchez, J.M., Navarro, L., 2016. Nectar robbing: a common phenomenon mainly determined by accessibility constraints, nectar volume and density of energy rewards. Oikos 125, 1044-1055.

Schaefer, H.M., Ruxton, G.D., 2011. Plant - Animal Communication Oxford. University Press, New York.

The plant list, 2013. Version 1.1, Published on the internet; http://www. theplantlist.org/ (accessed 30.07.16).

Thompson, J.N., 1982. Interaction and Coevolution. John Wiley, New York.

Valdivia, C.E., González-Gómez, P.L., 2006. A trade-off between the amount and distance of pollen dispersal triggered by the mixed foraging behaviour of Sephanoides sephaniodes (Trochilidae) on Lapageria rosea (Philesiaceae). Acta Oecol. 29, 324-327.

Vogel, S., 1990. The Role of Scent Glands in Pollination. Smithsonian Institute Library, Washington D.C.

Wang, Y., Wu, H., Sun, S., 2012. Persistence of pollination mutualisms in plant pollinator - robber systems. Theor. Popul. Biol. 81, 243-250.

Wang, Y., 2013. Dynamics of plant - pollinator - robber systems. J. Math. Biol. 66, $1155-1177$.

Willmer, P., 2011. Pollination and Floral Ecology. Princeton University Press, Princeton, New Jersey.

Wood, S.N., 2001. mgcv: GAMs and generalized ridge regression for R. R News 1, $20-25$.

Yanagizawa, Y.A.N.P., Maimoni-Rodella, R.C.S., 2007. Floral visitors and reproductive strategies in five melittophilous species of Bignoniaceae in Southeastern Brazil. Braz. Arch. Biol. Technol. 50, 1043-1050. 